\title{
Exosomes in Viral Disease
}

\author{
Monique R. Anderson ${ }^{1,2}$ • Fatah Kashanchi ${ }^{3}$ - Steven Jacobson ${ }^{1}$
}

Published online: 20 June 2016

(C) The American Society for Experimental NeuroTherapeutics, Inc. (outside the U.S.) 2016

\begin{abstract}
Viruses have evolved many mechanisms by which to evade and subvert the immune system to ensure survival and persistence. However, for each method undertaken by the immune system for pathogen removal, there is a counteracting mechanism utilized by pathogens. The new and emerging role of microvesicles in immune intercellular communication and function is no different. Viruses across many different families have evolved to insert viral components in exosomes, a subtype of microvesicle, with many varying downstream effects. When assessed cumulatively, viral antigens in exosomes increase persistence through cloaking viral genomes, decoying the immune system, and even by increasing viral infection in uninfected cells. Exosomes therefore represent a source of viral antigen that can be used as a biomarker for disease and targeted for therapy in the control and eradication of these disorders. With the rise in the persistence of new and reemerging viruses like Ebola and Zika, exploring the role of exosomes become more important than ever.
\end{abstract}

Electronic supplementary material The online version of this article (doi:10.1007/s13311-016-0450-6) contains supplementary material, which is available to authorized users.

Monique R. Anderson

andersonmr2@mail.nih.gov

1 National Institutes of Health, National Institute of Neurological Disorders and Stroke, Neuroimmunology Branch, Viral Immunology Section, Bethesda, MD 20892, USA

2 Department of Pathology Molecular and Cellular Basis of Disease Graduate Program, University of Virginia School of Medicine, Charlottesville, VA 22903, USA

3 George Mason University, National Center for Biodefense and Infectious Disease, Laboratory of Molecular Virology, Manassas, VA 20110, USA
Key Words Exosomes $\cdot$ microvesicles $\cdot$ multivesicular bodies · "back fusion" · ESCRT

\section{Introduction}

In the struggle to maintain health, the immune system has evolved several mechanisms to protect the host from pathogens. Foremost is the ability to differentiate self from pathogen, but tools are also required to mobilize components of the immune system in response to threats. Therefore, intercellular communication is key in this process. Until 30 years ago, cellto-cell signaling was encompassed by transfer of chemokines, cytokines, and direct cell contact. The discovery of vesicles being shed from the surface of reticulocytes as a mechanism for iron transfer forever changed how the field of immunology understood cellular communication [1,2]. Since then, the understanding of how microvesicles (MVs) contribute to immunity and pathogenicity has exploded. However, pathogens have evolved in concert with the immune system and have developed methods to subvert and co-opt this form of intercellular communication to evade the immune system and aid spread. The cross-over and interaction between MVs, and specifically exosomes, and viruses will be the subject of this review.

The term "microvesicles" (MVs) has undergone an evolution despite there being overwhelming evidence that their secretion is conserved across all kingdoms [3]. In preferred terminology, MVs include vesicles ranging in size from 100 to $1000 \mathrm{~nm}$ that are shed from the plasma membrane, as well as exosomes, which range in size from 30 to $100 \mathrm{~nm}$ and originate within microvesicular bodies (MVBs) [4, 5]. For the purposes of this review, we will focus our discussion on exosomes; however, there are several excellent reviews in the literature that address 
extracellular vesicles as a whole [6-11]. In appearance, exosomes are unilamellar vesicles composed of a lipid bilayer that have a homogenous cup-shaped appearance on scanning electromicroscopy [3, 12]. To date, they can be shown to originate from almost every cell type studied, including, but not limited to, T cells [13], B cells [14, 15], dendritic cells [16], neurons [17], astrocytes [18, 19], endothelial cells [20], smooth muscle cells [21], oligodendrocytes [22], and reticulocytes [2, 23]. The wide variety of cells that can excrete exosomes also dictates the wide array of materials in which they can be isolated: saliva, plasma, urine, cerebrospinal fluid, bronchial alveolar lavage, and serum [24]. The contents of exosomes are just as varied and depend heavily on the cells from which they originate but can be considered broadly to include proteins, mRNAs, microRNAs (miRNAs), lipids, and carbohydrates. Once released, the components within exosomes can influence the local microenvironment or spread through circulation to locations far removed from their origin. Indeed, exosomes have been shown to cross the blood-brain barrier [25], perhaps pointing to their role in allowing coordination with the immune system in even immune-privileged sites [26]. This aspect of exosome biology is especially important, as there are many instances in which viral proteins can be isolated, but the virus itself is difficult to detect. The presence of viral antigens in the absence of viral detection may be better understood through an understanding of exosome biogenesis and uptake.

\section{Exosome Biogenesis}

As aforementioned, exosomes originate in MVBs (late endosomes), which are a component of the endocytic pathway. MVBs contain intraluminal vesicles (ILVs) that range in size from 30 to $100 \mathrm{~nm}$ and can be targeted for 2 separate fates: lysosomal degradation or fusion with the plasma membrane, after which release of ILVs to the extracellular environment occurs and at which point they are termed exosomes [4, 27]. In a process called "back fusion", ILVs deliver plasma membrane invaginations (through clathrin-mediated and clathrin-independent endocytosis) to the endosomal network, making them (and therefore exosomes) capable of carrying both intracellular and extracellular materials [8, 28, 29]. Indeed, exosomes are seen as an exciting avenue for their capability in giving snapshots of the microenvironment, and serving as a good source of biomarkers. Part of this advantage is owing to the large cross-over between exosome biogenesis and egress, pathogen entry, and normal macromolecule entry and cycling within the cell $[23,27,28]$.
The exact intracellular signals that direct ILVs to the plasma membrane for release are still under investigation and will be discussed briefly here. Endosomal sorting complexes required for transport (ESCRT) machinery has been studied in its role for directing ubiquitin-labeled proteins into endosomes for delivery into MVBs, and, as such, ESCRT proteins, like Alix and TSG101, are enriched in exosomes [30, 31]. Lipid raft-associated proteins such as transferrin and caveolins, and other proteins involved in membrane trafficking, like the tetraspanins CD9, CD63, and CD81, which have been shown to bind to ESCRT machinery, are also enriched in exosomes [32-34]. There are now several databases that exist that list the assortment of materials that can be found in exosomes (Vesiclepedia, EVPedia, and Exocarta) [7, 35, 36].

However, ESCRT is not the only method by which exosome formation can occur as there are also ESCRTindependent methods by which proteins, lipids, and RNA can enter the endosomal pathway. For example, oligodendrocytes direct exosome formation via the ceramide pathway [37], while other cell types rely on oligomerization of tetraspanin complexes $[8,38,39]$. Furthermore, while knockdown of some ESCRT components may abrogate exosome production, it does not completely knock it out [40, 41]. Indeed, Rab GTPases, a known family of conserved proteins that regulate vesicular trafficking and membrane fusion events, are also involved in exosome formation as denoted by their high abundance in isolated exosomes [23, 42]. Several are implicated in the release of exosomes, including Rab11, Rab27, Rab5, Rab35, and Rab7, depending on cell type $[8,23]$. Rab 27a, in particular, regulates the fusion of MVBs at the plasma membrane to release ILVs [8, 23, 43]. Knockdown of Rab 27a inhibits exosome secretion from tumor cell lines $[4,43]$. There are several other Rabs that have also proven essential through a diminution in exosome levels after their knockdown, including Rab 2B, Rab9A, Rab5A, and Rab27b [4]. Being GTPases, the activation of each Rab is dependent on an influx of calcium, as is the case for Rab 11 in the K562 cell line, which may involve SNARE complexes [44-46]. Altogether, there are clearly several players within the cell that contribute to the endosomal compartment and, ultimately, to the release of exosomes, further emphasizing the importance of this pathway in normal biology. As such, it is not at all surprising that intracellular pathogens like viruses have also evolved mechanisms to incorporate their viral content at each stage of exosome formation.

\section{Fate of Exosomes}

Once in the extracellular space, exosomes can bind to neighboring cells, bind locally, travel passively through the bloodstream for a more paracrine destination, or be taken up by marginal zone phagocytes in the spleen and liver [4, 47]. 
Once released, they can have several fates, all of which dictate their downstream function. This can best be exemplified when analyzing the immune system and cancer cell biology, both of which have been extensively studied in the field of exosome biology. Importantly, the contents of exosomes have been shown to have activity in recipient cells [48], and are not just a means for removal of cellular waste material, as was first hypothesized, although this is still an important function [49]. First, exosomes can either be internalized or captured at the cell surface where they remain [4]. Both of these outcomes have been observed in dendritic cells [50]. Segura et al. [51] observed $\mathrm{CD}^{+}$dendritic cells capture exosomes via dendritic cell (DC) lymphocyte function-associated antigen 1 (LFA-1) at the surface for presentation of exosome-borne major histocompatibility complex (MHC)-peptide complexes to $\mathrm{CD} 4^{+} \mathrm{T}$ cells. These T cells could then be activated by exosomal antigens [52], a process that is much more efficient in the presence of DCs $[4,53,54]$. This process was dependent on intracellular adhesion molecule 1 (ICAM-1) expression on exosomes and is termed "cross-dressing" [55]. Another method by which DCs could capture exosomal antigens is through internalization, processing, and repackaging of endocytosed exosomal antigens into the endosomal pathway for representation on DC MHC II to naïve $\mathrm{CD}^{+} \mathrm{T}$ cells [50]. This process was documented in elegant experiments by Morelli et al. [50]. Activation of $\mathrm{CD}^{+} \mathrm{T}$ cells is also possible but requires the presence of DCs capable of "cross-presentation", that is, internalization of exosomal antigens followed by processing and representation on MHC I. This has been documented for the activation of tumor-specific cytotoxic $\mathrm{T}$ lymphocytes with tumor-derived exosomes [56]. Additionally, exosomes can be taken up by phagocytosis or pinocytosis where the contents can have direct consequences on gene expression of the recipient cells [57]. For example, regulatory $\mathrm{T}$ cells produce exosomes with miRNA profiles that directly downregulate inflammation in recipient cells [58]. Indeed, phagocytic uptake of exosomes can occur via phosphatidylserine on the surface of the vesicles via interaction with T-cell immunoglobulin and mucin domain-containing molecule (TIM)-4 on phagocytic cells [59, 60]. It has been shown that phagocytosis of exosomes from MT4, a human T-cell lymphotropic virus type I (HTLV-1) infected cell line, was reduced with the addition of TIM-4, but not TIM-1, antibodies [61]. This is intriguing as both TIM-1 and TIM-4 are known to serve has viral entry receptors for hepatitis A virus (HAV), Ebola, and several enveloped viruses $[62,63]$.

\section{Viral manipulation of Exosomal Pathway}

Remembering that exosomes originate in the endosomal compartment, viruses that enter via endocytosis are likely to co-opt exosomal communication; indeed, this proves to be the case.
Members of the Flaviviridae family, including important human pathogens like Dengue, West Nile virus (WNV), hepatitis $\mathrm{C}$ virus $(\mathrm{HCV})$, and the recently pandemic Zika virus, all enter via receptor-mediated or clathrin-mediated endocytosis [31, 64, 65]. HCV, Dengue, WNV and Zika virus then enter late endosomes after "back-fusion" of ILVs with this compartment, at which point the viral genome is dumped into the cytoplasm [66, 67]. However, in the case of $\mathrm{HCV}$, it is known that the viral genome can remain in ILVs and be secreted within exosomes, where they can operate as infectious particles [68, 69]. As noted in Table 1, there is very little difference in size between viruses and exosomes, making observations of infectivity from exosomes difficult to substantiate. Thus, to remove the possibility of viruses accounting for observed infections, Longatti et al. [69] utilized exosomes isolated from an $\mathrm{HCV}$ subgenomic replicon cell line, which lacks viral structural proteins and therefore cannot produce virions. By using a transwell assay, they showed that they were able to infect $\mathrm{Hu} 7$ cells after exposure to these shed exosomes without the need for direct cell-cell contact. Further, this infection was inhibited by an exosomal release inhibitor, a sphingomyelinase inhibitor. Thus far, HCV is the only member of the Flaviviridae family, known to incorporate genomic RNA into exosomes. Only hepatitis A virus (HAV), a nonenveloped picornavirus, is believed to use this mode of transmission as well [70]. However, it raises the possibility of other, yet unstudied, viruses utilizing the endosomal/ exosomal system as a means for delivery of viral message to uninfected cells. Indeed, this possibility was discussed in a review by Izquierdo et al. [71] of HIV and exosomes, and first dubbed the "Trojan Horse" hypothesis by Gould et al. in 2003 [72]. Gould et al. [72] hypothesize that with the similarities between HIV assembly and egress and exosome biogenesis, HIV has evolved to co-opt the exosome system and infect cells through packaging of the viral genome [72]. It is a theory supported by observations that HIV virions are released with exosomes and have enhanced infectivity in the presence of these vesicles [73]. However, this mechanism occurred via uptake of DCs, which subsequently transferred endocytosed HIV to closely associated uninfected T cells [74]. Specifically, HIV is endocytosed via DC sign into the endocytic pathway and trafficked back to the cell surface in intact DCs for presentation to T cells. However, direct packaging of HIV genome into exosomes has not been observed. Indeed, most

Table 1 Basic differences between exosomes and viruses

\begin{tabular}{lll}
\hline & Exosomes & Viruses \\
\hline Size & $30-100 \mathrm{~nm}$ & $2=0400 \mathrm{~nm}$ (depending on virus) \\
Shape & Uniform, cup-shaped & Variable \\
Charge & Usually lacking charge & Uniform, charged \\
\hline
\end{tabular}


research suggests that HIV budding is from the plasma membrane and not from the endosomal pathway $[75,76]$.

As mentioned, several viruses enter via the endocytic pathway. While some, like the flaviviruses, proceed through ILVs, some, like HIV, completely subvert this process and instead are closely associated with the ESCRT, lipid raft domains, and Rab GTPases that are involved in exosome trafficking [8]. For example, HIV Gag has been shown to interact with tetraspanins, especially CD63 and CD81, to aid in virion egress [77]. Human herpes virus (HHV)-6 virions have been visualized by electron microscopy in MVBs and egress by the exosomal release pathway [78]. The viral glycoprotein $\mathrm{gB}$ was shown to colocalize with $\mathrm{CD} 63$, although whether this relationship is necessary for association of HHV-6 virions and ILVs was unclear. However, HHV-6 infection dramatically increased MVB formation, indicating the endosomal/ exosomal pathway as important for HHV-6 infection and assembly [78]. Although the authors did not propose a mechanism by which viral components were incorporated in exosomes, the close association of the virus with exosomes in the endosomal pathway begs the question of whether HHV6 components are also packaged in exosomes, and requires further investigation.

Beyond interactions with tetraspanins, there are still other viruses that are capable of hitching onto the ESCRT complex to facilitate transportation of virus particles to the plasma membrane. For example, influenza A virus, respiratory syncytial virus (RSV), and certain Bunyaviruses attach to Rab1la vesicles to get to the plasma membrane for egress [8, 79-81]. Human cytomegalovirus (HCMV) is known to increase Rab27a, which, as noted earlier, is essential for MVB fusion with the plasma membrane [82]. The mechanism and ultimate change to exosome production is as yet unknown, but Rab27a could be found in association with its viral envelope [82]. HIV also interacts with Rab27a, and increases its levels, as well as those of several other components of the ESCRT machinery $[8,83-85]$. This is to be expected considering the aforementioned importance of ESCRT and exosome formation in HIV assembly and egress. Herpes simplex virus 1 (HSV1) is yet another virus that interacts with Rab27a. The interaction occurred through GSSHV-UL46, a viral tegument protein, in oligodendrocytes [86]. Viral glycoproteins $\mathrm{gH}$ and $\mathrm{gD}$ also interacted with Rab27a, and the importance of this association with Rab27a was proven in this paper by a decrease in viral production with Rab27a knockdown [86]. Whether this involved a decrease in exosome production, and how important this was for the lifecycle of HSV1, was not discussed at that time.

The movement of vesicles in the endosomal pathway as they track to and from the plasma membrane provides several opportunities for viral sabotage. Thus far, we have noted that viruses can enter the endosomal pathway just by viral fusion and entry into the cell. It was also noted that some viruses can hijack members of the ESCRT and vesicular trafficking machinery, and thereby integrate viral components into exosomes, owing to both close proximity and opportunity. This is important as endosomal trafficking allows for cellular contents, such as MHC II and tetraspanins to recycle through the cell and potentially incorporate other cellular proteins for representation at the plasma membrane. Thus, this process of "back fusion" may allow the entry of viral proteins that are being processed within the cell or even are targeted for the endosomal compartment. Additionally, viruses that require proximity to the nucleus and/or endoplasmic reticulum, such as herpesviruses and other DNA viruses, will also follow this route and thereby potentially have components incorporated into exosomes at this step [31, 87]. As stated, whether this process is natural happenstance due to proximity versus direct targeting is still under investigation but will be explored via the various examples mentioned herein.

\section{HIV}

HIV, which has been noted to take advantage of several steps in exosome biogenesis, has been shown to incorporate transactivating response (TAR) RNA into exosomes $[88,89]$. The uptake of exosomal TAR in recipient cells can downregulate apoptosis and is postulated to have a role in supporting HIV infection. Importantly, TAR RNA was still able to be detected in exosomes isolated from the serum of HIV-positive patients on highly active antiretroviral therapy, indicating that even with antiretroviral therapy, short transcripts remain present in these exosomes [90]. Indeed, the same group later went on to find that exosomal TAR RNA could stimulate proinflammatory cytokines in recipient cells through activation of the nuclear factor kappa b pathway [89]. In a separate study, the HIV Nef protein was found in released exosomes [91]. Later uptake of these $\mathrm{Nef}^{+}$exosomes led to increased susceptibility of naïve $\mathrm{T}$ cells to HIV infection [91, 92]. Indeed, another report found that exosomes from HIV-infected cells could reactivate HIV in latently infected cells [93]. Exosomal Nef has also been shown to increase T-cell apoptosis in vitro, which may contribute to the $\mathrm{CD} 4^{+}$T-cell depletion in AIDS pathogenesis [85]. Interestingly, Nef expression in $\mathrm{CD}^{+} \mathrm{T}$ cells was also noted to decrease CD4 and MHC I export to released exosomes [94]. The authors postulated that this decreases the ability of $\mathrm{CD}^{+} \mathrm{T}$ cells to inhibit HIV infection in uninfected cells by using exosomes as decoys to soak up HIV virions. This would further explain how $\mathrm{Nef}^{+}$exosomes enhance HIV infectivity. More recently, Luo et al. [95] dispute the incorporation of Nef into exosomes at all, despite several reports finding motifs within Nef that are necessary for exosomal incorporation [96, 97]. It is a subject that requires further investigation. Nevertheless, it is clear that HIV has 
evolved mechanisms to alter the cellular microenvironment to its advantage through exosomal cellular communication.

\section{HTLV}

Like HIV, there are several other viruses that also seemingly target viral RNAs and proteins for exosomal export. HTLV-1, another human retrovirus and the cause of adult T-cell leukemia and HTLV-1-associated myelopathy/tropical spastic paraparesis (HAM/TSP), has also been shown to incorporate viral proteins into shed exosomes. Jaworski et al. [98] found that HTLV-1-infected cell lines shed exosomes containing Tax, a pleiotropic transactivating protein implicated in the immune dysregulation associated with infection [99, 100]. Tax appears to be targeted for exosome entry by ubiquitination $[98,101]$, which was noted earlier to be an important target for ESCRT machinery. Indeed, prior studies have shown Tax colocalization with organelles undergoing exocytosis [102, 103].

Exosomes shed from $\mathrm{HTLV}-1^{+}$cell lines were found to also contain viral mRNA and miRNAs such as tax and $h b z$ [98]. Additionally, exosomes shed from HTLV-1-infected cell lines showed a different cell miRNA profile, as well as a unique set of host proteins and lipids, than was seen in those shed by uninfected cell lines. In as-yet-unpublished work from our laboratory in collaboration with Dr. Kashanchi, it was further demonstrated that HTLV-1 Tax could be found in exosomes isolated from the cerebrospinal fluid of some patients with HAM/TSP, while exosomes from uninfected controls were completely negative. Additionally, cultured, unstimulated peripheral blood mononuclear cells from patients with HAM/ TSP were shown to shed exosomes that contained Tax protein, as well as Tax mRNA. Unlike the cell lines, however, no detectable HBZ mRNA could be isolated from exosomes (unpublished data). This may have functional consequences as HTLV-1 is a cell-associated virus and shedding of viral antigens may contribute to the inflammatory immune response. Indeed, it has previously been shown that extracellular Tax can have damaging consequences for neurons [102, 104], although neither study specifically implicated exosomes. Once Tax protein or tax mRNA enter recipient cells, it can stimulate the production of proinflammatory cytokines, like interleukin (IL)-6 and tumor necrosis factor- $\alpha$ [105]. Importantly, initial results from our laboratory indicate that cells that take up these exosomes can become targets for lysis by HTLV-1-specific cytotoxic T cells.

Of interest in the pathogenesis of HAM/TSP is the lack of documented infection of resident neuronal cells. While astrocytes and microglia can be infected in vitro, they have not been shown to be infected in vivo [106]. Moreover, the loss of oligodendrocytes that occurs with disease progression cannot be explained by active infection. One postulation is that the proinflamatory environment contributes to their eventual breakdown, while others have proposed direct targeting of oligodendrocytes due to a mechanism of molecular mimicry of Tax to a neuronal protein [107-109]. Exosomal Tax may therefore ultimately explain both possibilities by contributing to the inflammatory cytokine production through packaging of these cytokines, as well as induction in recipient cells (Fig. 1). Additionally, exosomal Tax uptake in uninfected cells could explain targeting by HTLV-1-specific T cells in the presence of aseptic resident neuronal cells. Clearly, further research must be undertaken to further elucidate the role of exosomes in the disease progression of HAM/TSP and potential targeting of therapeutics.

\section{HSV1}

As mentioned, HSV1 is also known to interact with the ESCRT complex through viral tegument proteins and glycoproteins. Though this relationship with Rab27a has not yielded an observed consequence to exosomal production or content, viral proteins can also be found in exosomes shed from infected cells. HSV infection results in the release of a variety of MVs from the cell, most prominent of which are L particles, which are composed of virus envelope and tegument but lack viral genome and capsid proteins $[110,111]$. They are noninfectious on their own but have been shown to increase infectivity overall possibly through the transference of factors that increase susceptibility in uninfected cells [112, 113].

In contrast, exosomes shed from HSV1-infected cells express the tetraspanins known to be enriched on exosomes, CD9, CD63, and CD81 [114]. In addition to viral transcripts, HSV1 appears to direct the incorporation of certain host products into examples. An example of this is the Stimulator of INF genes (STING) protein. It was found not only to be upregulated with HSV infection in Vero cells, but also found to be shuttled out of cells in association with CD9 [115]. The authors demonstrated that STING was packaged in exosomes and delivered to uninfected cells. It was postulated that in addition to viral miRNAs also packaged in exosomes, such as miR-H5, miR-H3, and miR-H6, these exosomes could exert a negative effect on viral spread and potentially host-host infection by increasing host survival [114]. Conversely, immediate early transcripts such as ICP27 and late transcripts like VP16 can also be found in exosomes, thereby promoting spread and latency [115]. Additionally, HSV gB has been shown to colocalize with human leukocyte antigen-DR in exosomes shed from cells $[3,116]$. Shuttling of this MHC to exosomes reduced the ability of cells to present peptide to the immune system and was postulated to serve as important mechanism promoting viral latency. There is more work to be done in terms of characterizing the effects of packaging host and viral products into exosomes. More importantly, it would be of interest to 
Fig. 1 HTLV-1

Immunopathogenesis

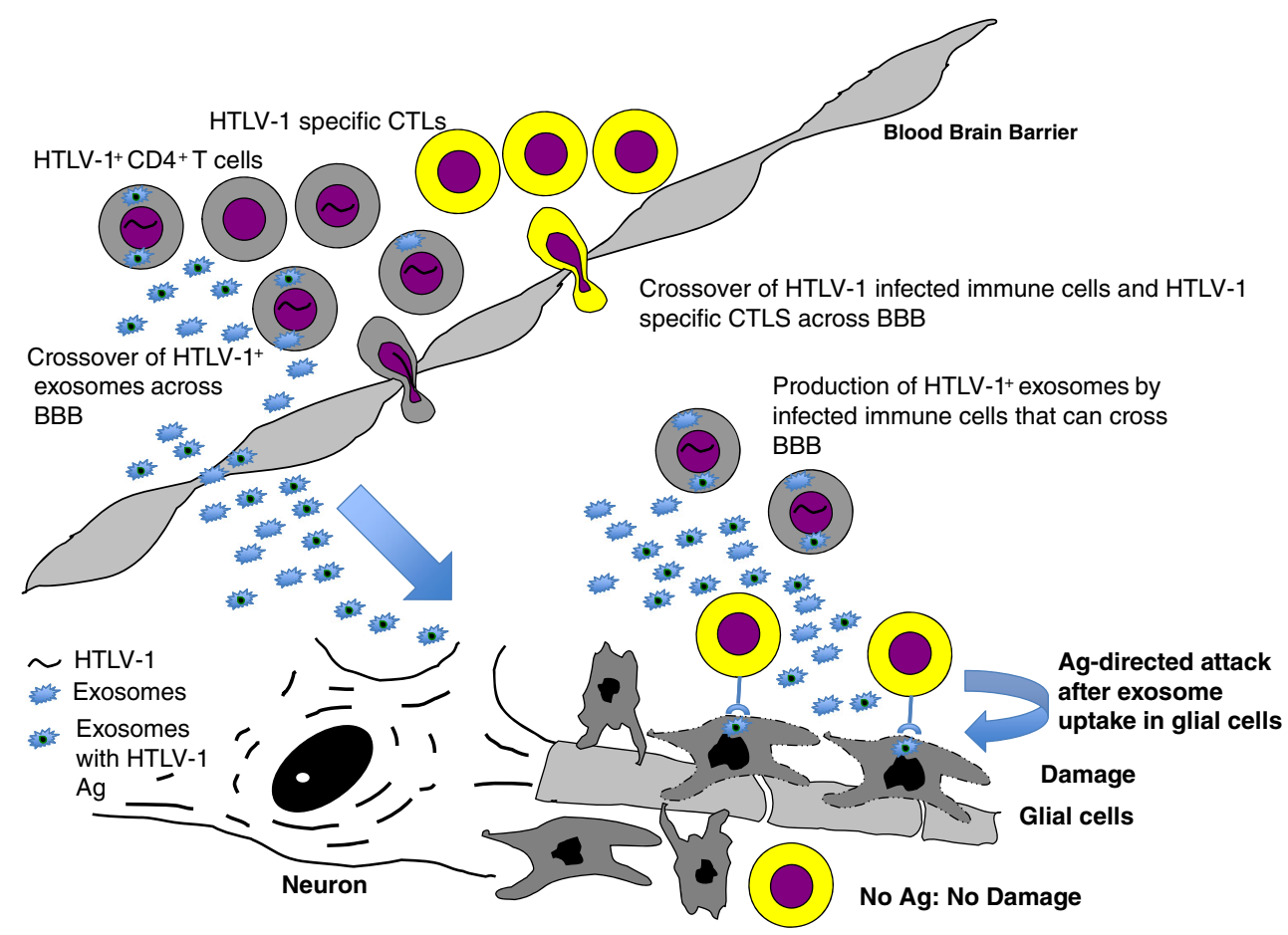

determine if patients that progress to central nervous system (CNS) disease or reactivation in the CNS have different exosome profiles compared with patients that remain latently infected. Of note, a literature search did not yield research on the association of exosomes with varicella zoster virus pathogenesis, although the role of exosomes in HSV1 pathogenesis suggests that additional research into this area would be warranted.

\section{Epstein-Barr Virus}

In addition to HSV1, several other herpesviruses also influence exosomal content. As mentioned, this may be owing to the proximity of herpes virus transcription with the ESCRT pathway as other cellular products enter via "back fusion" [31, 117]. Epstein-Barr virus (EBV) and Kaposi's sarcoma herpes virus (KSHV) have been the most studied, owing to the role of exosomes in the propogation of EBV- and KSHV-associated cancers, including nasopharyngeal sarcomas and Kaposi's sarcoma, respectively.

Latent membrane protein 1 (LMP1) was first discovered to be exported in exosomes isolated from $\mathrm{EBV}^{+}$cancer cells [118]. As a signal transduction protein, uptake of LMP1 $1^{+}$ exosomes was found to inhibit natural killer cell activity and T-cell activation and proliferation $[32,119,120]$. Additionally, similar to HSV1, EBV is able to promote the incorporation of host proteins into exosomes. Galectin-9 can be found in exosomes released from $\mathrm{EBV}^{+}$cells and can induce the apoptosis of EBV-specific T cells, signifying a method by which
EBV actively avoids detection by the immune system through exosomes [23, 121]. Furthermore, epidermal growth factor and fibroblast growth factor-2, both activated in association with LMP-1-mediated transformation, are shuttled into exosomes in LMP- $1^{+}$cells $[10,122]$. This may represent a mechanism by which EBV cancers alter the microenvironment to increase propagation and survival of transformed cells. Still further evidence exists for EBV promoting packaging of viral miRNAs. These miRNAs are then able to decrease target genes like CXCL11, which is an immunoregulatory gene important for antiviral activity [23]. EBV further evades immune detection by the shuttling of important immune effectors like IL-1 $\beta$, IL-18, and IL-33, into exosomes and out of the cell [123]. In this way, EBV also targets exosomes as a "trash receptacle". Taken together, all of these modulations to the exosomal pathway increase the survival and transformative abilities of EBV. Indeed, the transfer of viral antigens in the absence of active viral infection may lead to other consequences. A recent report by Baglio et al. [124] found that EBV EBER1, a viral small RNA, could be found in exosomes and that these exosomes, in turn, aberrantly activated DCs that were found in EBV-negative skin lesions of patients with lupus patients. This indicates a new potential mode of pathogenesis in autoimmune disease and other diseases where viral infection has been implicated, but a causal link has been difficult to verify. Furthermore, exosomes may serve as a potential tool for screening these affected individuals.

Another gamma herpes virus, KSHV or HHV-8, was also found to modulate the microenvironment through 
the packaging of viral factors in exosomes. Exosomes produced by infected cells were demonstrated to carry both KSHV and host-derived miRNA. Exposure to patientderived exosomes increased cell migration and IL- 6 production in recipient endothelial cells [125]. Additionally, like EBV, KSHV reprogrammed the cell profile of infected B cells [126]. Infection promoted a switch to glycolytic metabolism, and glycolysis by-products like pyruvate kinase and lactate dehydrogenase were packaged into exosomes [7, 127].

\section{CMV}

HHV-5, or CMV, is a betaherpesvirus. Like all of the herpes viruses, it is able to infect cells and establish latency. It is an especially important pathogen considered in transplant medicine, as CMV infection or CMV discordance between donor and recipient can lead to increased rates of organ rejection [128-130]. Previous reports have shown that infected epithelial cells can produce exosomes that stimulate allogeneic donor memory $\mathrm{CD}^{+} \mathrm{T}$ cells [20]. The authors proposed that this was a potential contributor to allograft rejection in transplant recipients. In addition to promoting an activated immune response, CMV infection can also increase the release of DCSIGN on exosomes in complex with the viral glycoprotein $\mathrm{gB}$ [131]. DC-SIGN is a C-type lectin family member that is necessary for virus uptake [132]. It was shown that this release mediated infection of myeloid DCs by CMV and increased overall CMV infectivity [131].

HHV-6, another betaherpesvirus, has already been discussed in terms of the viral association with the endosomal pathway and MVBs. Additionally, HHV-6 was later detected to increase MHC I transfer to released exosomes in addition to viral particles [133]. MHC I downregulation is a well-known mechanism for immunoevasion employed by many classes of pathogens. This insight, gained by studying HHV6, may point to shuttling of MHC I and MHC II to exosomes as an evolutionarily conserved mechanism that is used by many viruses.

\section{Human Papilloma Virus}

Exosome biology plays a large role in tumor pathogenesis. Thus, it is not surprising that so many oncogenic viruses have been shown to subjugate exosomal communication. Human papilloma virus (HPV) has several different serotypes, some of which are more associated with cervical cancer-like HPV type 16 and 18. In investigating patients with tumorigenic versus benign subtypes, differential miRNA content in exosomes was noted. Indeed, the miRNA profile of exosomes isolated from cells infected with pathogenic HPV strains were enriched for miRNA that controlled cell proliferation and apoptosis [134]. Although there was no evidence for the incorporation of viral oncogenes E6/E7 in released exosomes, the levels of E6/E7 were important in dictating the miRNA profile of released exosomes $[8,135]$. Owing to this discrepancy in the profile of exosomes shed from oncogenic versus benign strains, there is a clinical trial currently underway at the National Institutes of Health to investigate the utility of screening salivary exosomes in patients at risk for HPV oropharyngeal carcinoma (https://clinicaltrials.gov/ct2 /show/NCT02147418). Indeed, the importance of exosomes has been reinforced by a recent study in which normal cells were immortalized after incubation with exosomes derived from breast cancer cells [136].

\section{HAV}

HAV is a nonenveloped picorna virus that usually causes a mild gastrointestinal illness but which can, at times, lead to hepatitis with full clearance of the virus after infection. Interestingly, HAV has been shown to hijack endosomal membranes for encapsulation upon release [137]. These enveloped viruses are fully infectious and aid HAV in immunoevasion as they are protected from antibody neutralization [138]. Although envelopment of previously naked virus particles is different to the strategy taken by $\mathrm{HCV}$, which incorporates naked viral genome into exosomes, both HAV and HCV coopt the exosomal system for evasion from the adaptive arm of the immune system. Indeed, hepatitis B virus, which is a hepadnavirus, also hijacks exosomal communication. On HBV infection, cellular release of MVs is dramatically increased. However, these MVs are characterized as subviral particles and contain viral surface antigens but no viral genome and are therefore noninfectious [139]. The subviral particles can, however, serve as decoys to the immune system as they are able to absorb antibodies that might otherwise inhibit viral particles [32].

\section{Bunyaviruses}

Bunyaviridae is a family of enveloped, negative-stranded viruses that includes the hantavirus, nairovirus, orthobunyavirus, phlebovirus, and tospovirus genera. Of these, hantavirus and phlebovirus have clinical significance as human pathogens, including hanta pulmonary virus and Rift Valley fever virus (RVFV), respectively. Interestingly, recent reports suggest that the phlebovirus family can modulate the immune system via exosomes. Exosomes isolated from cells infected with RVFV not only contained viral RNA and proteins, but also immune cells exposed to these exosomes underwent apoptosis [140]. This suggests a mechanism for immune persistence early in the disease, as exosomes were detected prior to virion release [140]. 
Another phelebovirus, a newly recognized virus called severe fever with thrombocytopenia syndrome virus, also utilizes exosomes. Severe fever with thrombocytopenia syndrome virus was found to package virions within $\mathrm{CD}^{+} 3^{+}$extracellular vesicles (exosomes), which allowed for efficient receptor-independent uptake by neighboring cells [141]. Of note, pheleboviruses also have DC-SIGN as a receptor [142], which has been shown to be packaged into exosomes for uptake in uninfected cells by CMV [131]. Further investigation should be undertaken to observe if a similar mechanism for viral spread is employed by phleboviruses and bunyaviruses in general. There is currently no literature describing hantaviruses and exosomes, although this topic obviously warrants further investigation for the role of extracellular vesicles in Hanta pulmonary virus syndrome.

\section{Discussion}

As can be seen by the wide variety of viruses discussed in this review, there are several different viruses spread across different genera that take advantage of exosomal communication. While some cloak viral antigens to aid in viral spread, others increase shuttling of disadvantageous host products that would hinder viral infection into exosomes. Others utilize exosomes as decoys to the immune system and, counterintuitively, some viruses also seem to prime a directed immune response to inhibit spread. The examples presented by these viruses illustrate the many ways exosomal communication is important for immune function and maintenance of the cellular microenvironment. The one unifying factor is that all of the viruses present, except for HAV and the phleboviruses, are able to establish latency in the host. Thus, the question becomes: Does their ability to manipulate exosomal contents allow these viruses to establish latency, or does long-term infection favor the ability to isolate exosomes while transient infections have been largely ignored due to the belief that immune clearance negates the risk of exosomes? Indeed, the example of RVFV indicates that exosomes may play an early role in the viral spread of transient infections. Of note, the line between latency and transient infection is becoming blurred in the cases of Zika and Ebola, which have shown a remarkable ability to reappear in previously convalescent patients. Furthermore, the examples in this review highlight the ability of exosomes to increase not only viral spread, but also the type of cells that can be infected. This becomes an important consideration as we attempt to understand how viruses are able to establish and maintain viral reservoirs and "pockets of infection" in cells not normally believed to be permissive.

Ebola, chikungunya virus, avian flu, and Zika virus are just a few of the new and re-emerging viruses that are characterized by transient infection. However, in the cases of Ebola and Zika virus, scientists are discovering that virus and viral proteins can still be isolated months after the initial infection from immune privileged sites like the eye and semen [143-145]. All of these viruses pose serious health threats to the human population, and both Zika and Ebola have been of concern owing to increasing evidence of CNS disease [146-148]. Thus, an understanding of how these agents persist in these spaces and cause damage is imperative. As noted in this review, exosomes are a potential source of antigen that can easily cross the endothelial barriers protecting immune privileged sites. Additionally, in the case of Zika virus, which is a flavivirus, there is already precedence for other members of this virus family altering exosomal contents. Therefore, there is a high likelihood that this ability to incorporate viral contents into exosomes is a shared mechanism for immune evasion amongst the entire family, and warrants investigation. Considering the troubling consequences of Zika infection in pregnancy, screening for exosomes in prospective mothers might be a useful tool for evaluation of risks to the fetus. This is an attractive prospect as the ability to detect Zika virus by reverse transcription polymerase chain reaction is optimal 5-7 days after symptom onset and while the patient is still viremic (http://www.cdc. gov/zika/hc-providers/qa-pregnant-women.html). Additional testing involves detection of $\operatorname{IgM}$; however, there is crossreactivity with other flaviviruses like Dengue virus and $\mathrm{WNV}$, and therefore it is not specific. A method that targets particles, such as exosomes, that can cross the placenta and that could still be detected after the window of viremia would be useful. Exosomes represent a potential biomarker that can be used in this way. Furthermore, exosome analysis may prove to be important in understanding Ebola pathophysiology as Alix, the ESCRT protein important in directing proteins to endosomes, is necessary for viral budding [149].

Overall, exosomes represent an exciting new avenue with which to explore in viral pathology, one that current evidence suggests plays a role in both transient and latent virus infection. The possibility of screening exosomes in viral disease presents a noninvasive technique for the management and treatment of patients.

Required Author Forms Disclosure forms provided by the authors are available with the online version of this article.

\section{References}

1. Trams EG, Lauter CJ, Salem N, Jr., Heine U. Exfoliation of membrane ecto-enzymes in the form of micro-vesicles. Biochim Biophys Acta 1981;645(1):63-70.

2. Johnstone RM, Bianchini A, Teng K. Reticulocyte maturation and exosome release: transferrin receptor containing exosomes shows multiple plasma membrane functions. Blood 1989;74(5):18441851. 
3. Lai FW, Lichty BD, Bowdish DM. Microvesicles: ubiquitous contributors to infection and immunity. J Leukoc Biol 2015;97(2):237-245.

4. Robbins PD, Morelli AE. Regulation of immune responses by extracellular vesicles. Nat Rev Immunol 2014;14(3):195-208.

5. van der Pol E, Boing AN, Harrison P, Sturk A, Nieuwland R. Classification, functions, and clinical relevance of extracellular vesicles. Pharmacol Rev 2012;64(3):676-705.

6. van Dongen HM, Masoumi N, Witwer KW, Pegtel DM. Extracellular vesicles exploit viral entry routes for cargo delivery. Microbiol Mol Biol Rev 2016;80(2):369-386.

7. Schwab A, Meyering SS, Lepene B, et al. Extracellular vesicles from infected cells: potential for direct pathogenesis. Front Microbiol 2015;6:1132.

8. Alenquer M, Amorim MJ. Exosome biogenesis, regulation, and function in viral infection. Viruses 2015;7(9):5066-5083.

9. Chahar HS, Bao X, Casola A. Exosomes and their role in the life cycle and pathogenesis of RNA viruses. Viruses 2015;7(6):32043225.

10. Meckes DG, Jr. Exosomal communication goes viral. J Virol 2015;89(10):5200-5203.

11. Wurdinger T, Gatson NN, Balaj L, Kaur B, Breakefield XO, Pegtel DM. Extracellular vesicles and their convergence with viral pathways. Adv Virol 2012;2012:767694.

12. Li XB, Zhang ZR, Schluesener HJ, Xu SQ. Role of exosomes in immune regulation. J Cell Mol Med 2006;10(2):364-375.

13. Blanchard N, Lankar D, Faure F, et al. TCR activation of human T cells induces the production of exosomes bearing the TCR/CD3/ zeta complex. J Immunol 2002;168(7):3235-3241.

14. Raposo G, Nijman HW, Stoorvogel W, et al. B lymphocytes secrete antigen-presenting vesicles. J Exp Med 1996;183(3):11611172.

15. Escola JM, Kleijmeer MJ, Stoorvogel W, Griffith JM, Yoshie O, Geuze HJ. Selective enrichment of tetraspan proteins on the internal vesicles of multivesicular endosomes and on exosomes secreted by human B-lymphocytes. J Biol Chem 1998;273(32):2012120127.

16. Thery C, Regnault A, Garin J, P et al. Molecular characterization of dendritic cell-derived exosomes. Selective accumulation of the heat shock protein hsc73. J Cell Biol 1999;147(3):599-610.

17. Faure J, Lachenal G, Court M, et al. Exosomes are released by cultured cortical neurones. Mol Cell Neurosci 2006;31(4):642648.

18. Basso M, Bonetto V. Extracellular vesicles and a novel form of communication in the brain. Front Neurosci 2016;10:127.

19. Wang G, Dinkins M, He Q, et al. Astrocytes secrete exosomes enriched with proapoptotic ceramide and prostate apoptosis response 4 (PAR-4): potential mechanism of apoptosis induction in Alzheimer disease (AD). J Biol Chem 2012;287(25):2138421395.

20. Walker JD, Maier CL, Pober JS. Cytomegalovirus-infected human endothelial cells can stimulate allogeneic CD4+ memory T cells by releasing antigenic exosomes. J Immunol 2009;182(3):15481559 .

21. Comelli L, Rocchiccioli S, Smirni S, et al. Characterization of secreted vesicles from vascular smooth muscle cells. Mol Biosyst 2014;10(5):1146-1152.

22. Kramer-Albers EM, Bretz N, Tenzer S, et al. Oligodendrocytes secrete exosomes containing major myelin and stress-protective proteins: Trophic support for axons? Proteomics Clin Appl 2007;1(11):1446-1461.

23. Schorey JS, Cheng Y, Singh PP, Smith VL. Exosomes and other extracellular vesicles in host-pathogen interactions. EMBO Rep 2015;16(1):24-43.
24. Boukouris S, Mathivanan S. Exosomes in bodily fluids are a highly stable resource of disease biomarkers. Proteomics Clin Appl 2015;9(3-4):358-367.

25. Yang T, Martin P, Fogarty B, et al. Exosome delivered anticancer drugs across the blood-brain barrier for brain cancer therapy in Danio rerio. Pharm Res 2015;32(6):2003-2014.

26. El Andaloussi S, Lakhal S, Mager I, Wood MJ. Exosomes for targeted siRNA delivery across biological barriers. Adv Drug Deliv Rev 2013;65(3):391-397.

27. Murk JL, Stoorvogel W, Kleijmeer MJ, Geuze HJ. The plasticity of multivesicular bodies and the regulation of antigen presentation. Semin Cell Develop Biol 2002;13(4):303-311.

28. Bissig C, Gruenberg J. ALIX and the multivesicular endosome: ALIX in Wonderland. Trends Cell Biol 2014;24(1):19-25.

29. Pegtel DM, van de Garde MD, Middeldorp JM. Viral miRNAs exploiting the endosomal-exosomal pathway for intercellular cross-talk and immune evasion. Biochim Biophys Acta 2011;1809(11-12):715-721.

30. Matsuo H, Chevallier J, Mayran N, et al. Role of LBPA and Alix in multivesicular liposome formation and endosome organization. Science 2004;303(5657):531-534.

31. Nour AM, Modis Y. Endosomal vesicles as vehicles for viral genomes. Trends Cell Biol 2014;24(8):449-454.

32. Meckes DG, Jr., Raab-Traub N. Microvesicles and viral infection. J Virol 2011;85(24):12844-12854.

33. Gan X, Gould SJ. Identification of an inhibitory budding signal that blocks the release of HIV particles and exosome/microvesicle proteins. Mol Biol Cell 2011;22(6):817-830.

34. Sampey GC, Meyering SS, Asad Zadeh M, Saifuddin M, Hakami RM, Kashanchi F. Exosomes and their role in CNS viral infections. J Neurovirol 2014;20(3):199-208.

35. Kalra H, Simpson RJ, Ji H, et al. Vesiclepedia: a compendium for extracellular vesicles with continuous community annotation. PLoS Biol 2012;10(12):e1001450.

36. Kim DK, Kang B, Kim OY, et al. EVpedia: an integrated database of high-throughput data for systemic analyses of extracellular vesicles. J Extracell Vesicles 2013;2.

37. Trajkovic K, Hsu C, Chiantia S, et al. Ceramide triggers budding of exosome vesicles into multivesicular endosomes. Science 2008;319(5867):1244-1247.

38. van Niel G, Charrin S, Simoes S, et al. The tetraspanin CD63 regulates ESCRT-independent and -dependent endosomal sorting during melanogenesis. Develop Cell 2011;21(4):708-721.

39. Perez-Hernandez D, Gutierrez-Vazquez C, Jorge I, et al. The intracellular interactome of tetraspanin-enriched microdomains reveals their function as sorting machineries toward exosomes. J Biol Chem 2013;288(17):11649-11661.

40. Stuffers S, Sem Wegner C, Stenmark H, Brech A. Multivesicular endosome biogenesis in the absence of ESCRTs. Traffic 2009;10(7):925-937.

41. Tamai K, Tanaka N, Nakano T, et al. Exosome secretion of dendritic cells is regulated by Hrs, an ESCRT- 0 protein. Biochem Biophys Res Commun 2010;399(3):384-390.

42. Schwartz SL, Cao C, Pylypenko O, Rak A, Wandinger-Ness A. Rab GTPases at a glance. J Cell Sci 2007;120(Pt 22):3905-3910.

43. Ostrowski M, Carmo NB, Krumeich S, et al. Rab27a and Rab27b control different steps of the exosome secretion pathway. Nat Cell Biol 2010;12(1):19-30.

44. Savina A, Fader CM, Damiani MT, Colombo MI. Rab11 promotes docking and fusion of multivesicular bodies in a calciumdependent manner. Traffic 2005;6(2):131-143.

45. Colombo M, Raposo G, Thery C. Biogenesis, secretion, and intercellular interactions of exosomes and other extracellular vesicles. Annu Rev Cell Develop Biol 2014;30:255-289.

46. Fader CM, Sanchez DG, Mestre MB, Colombo MI. TI-VAMP/ VAMP7 and VAMP3/cellubrevin: two v-SNARE proteins 
involved in specific steps of the autophagy/multivesicular body pathways. Biochim Biophys Acta 2009;1793(12):1901-1916.

47. Montecalvo A, Shufesky WJ, Stolz DB, et al. Exosomes as a short-range mechanism to spread alloantigen between dendritic cells during $\mathrm{T}$ cell allorecognition. J Immunol 2008;180(5): 3081-3090

48. Lagana A, Russo F, Veneziano D, et al. Extracellular circulating viral microRNAs: current knowledge and perspectives. Front Genet 2013;4:120.

49. Thebaud B, Stewart DJ. Exosomes: cell garbage can, therapeutic carrier, or trojan horse? Circulation 2012;126(22):2553-2555.

50. Morelli AE, Larregina AT, Shufesky WJ, et al. Endocytosis, intracellular sorting, and processing of exosomes by dendritic cells. Blood 2004;104(10):3257-3266

51. Segura E, Guerin C, Hogg N, Amigorena S, Thery C. CD8+ dendritic cells use LFA-1 to capture MHC-peptide complexes from exosomes in vivo. J Immunol 2007;179(3):1489-1496.

52. Thery C, Duban L, Segura E, Veron P, Lantz O, Amigorena S. Indirect activation of naive $\mathrm{CD} 4+\mathrm{T}$ cells by dendritic cell-derived exosomes. Nat Immunol 2002;3(12):1156-1162.

53. Vincent-Schneider H, Stumptner-Cuvelette P, Lankar D, et al. Exosomes bearing HLA-DR1 molecules need dendritic cells to efficiently stimulate specific T cells. Int Immunol 2002;14(7): 713-722.

54. Admyre C, Johansson SM, Paulie S, Gabrielsson S. Direct exosome stimulation of peripheral human $\mathrm{T}$ cells detected by ELISPOT. Eur J Immunol 2006;36(7):1772-1781.

55. Segura E, Nicco C, Lombard B, et al. ICAM-1 on exosomes from mature dendritic cells is critical for efficient naive T-cell priming. Blood 2005;106(1):216-223.

56. Wolfers J, Lozier A, Raposo G, et al. Tumor-derived exosomes are a source of shared tumor rejection antigens for CTL cross-priming. Nat Med 2001;7(3):297-303

57. Mulcahy LA, Pink RC, Carter DR. Routes and mechanisms of extracellular vesicle uptake. J Extracell Vesicles 2014;3.

58. Okoye IS, Coomes SM, Pelly VS, et al. MicroRNA-containing Tregulatory-cell-derived exosomes suppress pathogenic $\mathrm{T}$ helper 1 cells. Immunity 2014;41(1):89-103.

59. Zakharova L, Svetlova M, Fomina AF. T cell exosomes induce cholesterol accumulation in human monocytes via phosphatidylserine receptor. J Cell Physiol 2007;212(1):174-181.

60. Miyanishi M, Tada K, Koike M, Uchiyama Y, Kitamura T, Nagata S. Identification of Tim4 as a phosphatidylserine receptor. Nature 2007;450(7168):435-439.

61. Feng D, Zhao WL, Ye YY, et al. Cellular internalization of exosomes occurs through phagocytosis. Traffic 2010;11(5):675687.

62. Moller-Tank S, Maury W. Phosphatidylserine receptors: enhancers of enveloped virus entry and infection. Virology 2014:468-470:565-80.

63. Rhein BA, Brouillette RB, Schaack GA, Chiorini JA, Maury W. Characterization of human and murine T-cell immunoglobulin mucin domain 4 (TIM-4) IgV domain residues critical for Ebola virus entry. J Virol 2016 Apr 27.

64. Smit JM, Moesker B, Rodenhuis-Zybert I, Wilschut J. Flavivirus cell entry and membrane fusion. Viruses 2011;3(2):160-171.

65. Hamel R, Dejarnac O, Wichit S, et al. Biology of Zika virus infection in human skin cells. J Virol 2015;89(17):8880-8896.

66. Nour AM, Li Y, Wolenski J, Modis Y. Viral membrane fusion and nucleocapsid delivery into the cytoplasm are distinct events in some flaviviruses. PLoS Pathog 2013;9(9):e1003585.

67. Cohen FS. How viruses invade cells. Biophys J 2016;110(5): 1028-1032.

68. Ramakrishnaiah V, Thumann C, Fofana I, et al. Exosomemediated transmission of hepatitis $\mathrm{C}$ virus between human hepatoma Huh7.5 cells. Proc Natl Acad Sci U S A 2013;110(32):13109-13113.

69. Longatti A, Boyd B, Chisari FV. Virion-independent transfer of replication-competent hepatitis $\mathrm{C}$ virus RNA between permissive cells. J Virol 2015;89(5):2956-2961.

70. Longatti $\mathrm{A}$. The dual role of exosomes in hepatitis $\mathrm{A}$ and $\mathrm{C}$ virus transmission and viral immune activation. Viruses 2015;7(12): 6707-6715.

71. Izquierdo-Useros N, Naranjo-Gomez M, Erkizia I, et al. HIV and mature dendritic cells: Trojan exosomes riding the Trojan horse? PLoS Pathog 2010;6(3):e1000740.

72. Gould SJ, Booth AM, Hildreth JE. The Trojan exosome hypothesis. Proc Natl Acad Sci U S A 2003;100(19):10592-10597.

73. Wiley RD, Gummuluru S. Immature dendritic cell-derived exosomes can mediate HIV-1 trans infection. Proc Natl Acad Sci U S A 2006;103(3):738-743.

74. Piguet V, Steinman RM. The interaction of HIV with dendritic cells: outcomes and pathways. Trends Immunol 2007;28(11): 503-510.

75. Yandrapalli N, Muriaux D, Favard C. Lipid domains in HIV-1 assembly. Front Microbiol 2014;5:220.

76. Woodward CL, Cheng SN, Jensen GJ. Electron cryotomography studies of maturing HIV-1 particles reveal the assembly pathway of the viral core. J Virol 2015;89(2):1267-1277.

77. Madison MN, Okeoma CM. Exosomes: implications in HIV-1 pathogenesis. Viruses 2015;7(7):4093-4118.

78. Mori Y, Koike M, Moriishi E, et al. Human herpesvirus-6 induces MVB formation, and virus egress occurs by an exosomal release pathway. Traffic 2008;9(10):1728-1742.

79. Bruce EA, Digard P, Stuart AD. The Rab11 pathway is required for influenza A virus budding and filament formation. J Virol 2010;84(12):5848-5859.

80. Rowe RK, Suszko JW, Pekosz A. Roles for the recycling endosome, Rab8, and Rab11 in hantavirus release from epithelial cells. Virology 2008;382(2):239-249.

81. Utley TJ, Ducharme NA, Varthakavi V, et al. Respiratory syncytial virus uses a Vps4-independent budding mechanism controlled by Rab11-FIP2. Proc Natl Acad Sci U S A 2008;105(29):1020910214.

82. Fraile-Ramos A, Cepeda V, Elstak E, van der Sluijs P. Rab27a is required for human cytomegalovirus assembly. PLoS One 2010;5(12):e15318.

83. Gerber PP, Cabrini M, Jancic C, et al. Rab27a controls HIV-1 assembly by regulating plasma membrane levels of phosphatidylinositol 4,5-bisphosphate. J Cell Biol 2015;209(3): 435-452.

84. Meng B, Ip NC, Prestwood LJ, Abbink TE, Lever AM. Evidence that the endosomal sorting complex required for transport-II (ESCRT-II) is required for efficient human immunodeficiency virus-1 (HIV-1) production. Retrovirology 2015;12:72.

85. Lenassi M, Cagney G, Liao M, et al. HIV Nef is secreted in exosomes and triggers apoptosis in bystander $\mathrm{CD} 4+\mathrm{T}$ cells. Traffic 2010;11(1):110-122

86. Bello-Morales R, Crespillo AJ, Fraile-Ramos A, Tabares E, Alcina A, Lopez-Guerrero JA. Role of the small GTPase Rab27a during herpes simplex virus infection of oligodendrocytic cells. BMC Microbiol 2012;12:265.

87. Krummenacher C, Carfi A, Eisenberg RJ, Cohen GH. Entry of herpesviruses into cells: the enigma variations. Adv Exp Med Biol 2013;790:178-195

88. Narayanan A, Iordanskiy S, Das R, et al. Exosomes derived from HIV-1-infected cells contain trans-activation response element RNA. J Biol Chem 2013;288(27):20014-20033.

89. Sampey GC, Saifuddin M, Schwab A, et al. Exosomes from HIV1 -infected cells stimulate production of pro-inflammatory 
cytokines through trans-activating response (TAR) RNA. J Biol Chem 2016;291(3):1251-1266.

90. Jaworski E, Saifuddin M, Sampey G, et al. The use of Nanotrap particles technology in capturing HIV-1 virions and viral proteins from infected cells. PLoS One 2014;9(5):e96778.

91. Campbell TD, Khan M, Huang MB, Bond VC, Powell MD. HIV$1 \mathrm{Nef}$ protein is secreted into vesicles that can fuse with target cells and virions. Ethn Dis 2008;18(2 Suppl. 2):S2-14-9.

92. Arenaccio C, Chiozzini C, Columba-Cabezas S, et al. Exosomes from human immunodeficiency virus type 1 (HIV-1)-infected cells license quiescent CD4+ T lymphocytes to replicate HIV-1 through a Nef- and ADAM17-dependent mechanism. J Virol 2014;88(19):11529-11539.

93. Arenaccio C, Anticoli S, Manfredi F, Chiozzini C, Olivetta E, Federico M. Latent HIV-1 is activated by exosomes from cells infected with either replication-competent or defective HIV-1. Retrovirology 2015;12:87.

94. de Carvalho JV, de Castro RO, da Silva EZ, et al. Nef neutralizes the ability of exosomes from CD4+ T cells to act as decoys during HIV-1 infection. PLoS One 2014;9(11):e113691.

95. Luo X, Fan Y, Park IW, He JJ. Exosomes are unlikely involved in intercellular Nef transfer. PLoS One 2015;10(4):e0124436.

96. Ali SA, Huang MB, Campbell PE, et al. Genetic characterization of HIV type 1 Nef-induced vesicle secretion. AIDS Res Hum Retroviruses 2010;26(2):173-192.

97. Campbell PE, Isayev O, Ali SA, et al. Validation of a novel secretion modification region (SMR) of HIV-1 Nef using cohort sequence analysis and molecular modeling. J Mol Model 2012;18(10):4603-4613.

98. Jaworski E, Narayanan A, Van Duyne R, et al. Human Tlymphotropic virus type 1-infected cells secrete exosomes that contain Tax protein. J Biol Chem 2014;289(32):22284-22305.

99. Romanelli MG, Diani E, Bergamo E, et al. Highlights on distinctive structural and functional properties of HTLV Tax proteins. Front Microbiol 2013;4:271.

100. Currer R, Van Duyne R, Jaworski E, et al. HTLV tax: a fascinating multifunctional co-regulator of viral and cellular pathways. Front Microbiol 2012;3:406.

101. Shembade N, Harhaj EW. Role of post-translational modifications of HTLV-1 Tax in NF-kappaB activation. World J Biol Chem 2010;1(1):13-20.

102. Alefantis T, Mostoller K, Jain P, Harhaj E, Grant C, Wigdahl B. Secretion of the human T cell leukemia virus type I transactivator protein tax. J Biol Chem 2005;280(17):17353-17362.

103. Alefantis T, Jain P, Ahuja J, Mostoller K, Wigdahl B. HTLV-1 Tax nucleocytoplasmic shuttling, interaction with the secretory pathway, extracellular signaling, and implications for neurologic disease. J Biomed Sci 2005;12(6):961-974.

104. Cowan EP, Alexander RK, Daniel S, Kashanchi F, Brady JN. Induction of tumor necrosis factor alpha in human neuronal cells by extracellular human T-cell lymphotropic virus type 1 Tax. J Virol 1997;71(9):6982-6989.

105. Dhib-Jalbut S, Hoffman PM, Yamabe T, et al. Extracellular human T-cell lymphotropic virus type I Tax protein induces cytokine production in adult human microglial cells. Ann Neurol 1994;36(5): 787-790.

106. Lepoutre V, Jain P, Quann K, Wigdahl B, Khan ZK. Role of resident CNS cell populations in HTLV-1-associated neuroinflammatory disease. Front Biosci 2009;14:1152-1168.

107. Kubota R, Nagai M, Kawanishi T, Osame M, Jacobson S. Increased HTLV type 1 tax specific CD8+ cells in HTLV type 1-asociated myelopathy/tropical spastic paraparesis: correlation with HTLV type 1 proviral load. AIDS Res Hum Retroviruses 2000;16(16):1705-1709.

108. Levin MC, Lee SM, Morcos Y, Brady J, Stuart J. Cross-reactivity between immunodominant human T lymphotropic virus type I tax and neurons: implications for molecular mimicry. J Infect Dis 2002;186(10):1514-1517.

109. Irish BP, Khan ZK, Jain P, et al. Molecular mechanisms of neurodegenerative diseases induced by human retroviruses: a review. Am J Infect Dis 2009;5(3):231-258.

110. Rixon FJ, Addison C, McLauchlan J. Assembly of enveloped tegument structures (L particles) can occur independently of virion maturation in herpes simplex virus type 1-infected cells. J Gen Virol 1992;73(Pt 2):277-284.

111. McLauchlan J, Rixon FJ. Characterization of enveloped tegument structures (L particles) produced by alphaherpesviruses: integrity of the tegument does not depend on the presence of capsid or envelope. J Gen Virol 1992;73(Pt 2):269-276.

112. McLauchlan J, Addison C, Craigie MC, Rixon FJ. Noninfectious L-particles supply functions which can facilitate infection by HSV-1. Virology 1992;190(2):682-688.

113. Heilingloh CS, Kummer M, Muhl-Zurbes P, et al. L particles transmit viral proteins from herpes simplex virus 1-infected mature dendritic cells to uninfected bystander cells, inducing CD83 downmodulation. J Virol 2015;89(21):11046-11055.

114. Kalamvoki M, Deschamps T. Extracellular vesicles during Herpes Simplex Virus type 1 infection: an inquire. Virol J 2016;13(1):63.

115. Kalamvoki M, Du T, Roizman B. Cells infected with herpes simplex virus 1 export to uninfected cells exosomes containing STING, viral mRNAs, and microRNAs. Proc Natl Acad Sci U S A 2014;111(46):E4991-E4996.

116. Temme S, Eis-Hubinger AM, McLellan AD, Koch N. The herpes simplex virus-1 encoded glycoprotein B diverts HLA-DR into the exosome pathway. J Immunol. 2010;184(1):236-243.

117. Lee CP, Liu PT, Kung HN, et al. The ESCRT machinery is recruited by the viral BFRF1 protein to the nucleus-associated membrane for the maturation of Epstein-Barr Virus. PLoS Pathog 2012;8(9): e1002904.

118. Flanagan J, Middeldorp J, Sculley T. Localization of the EpsteinBarr virus protein LMP 1 to exosomes. J Gen Virol 2003;84(Pt 7): 1871-1879.

119. Dukers DF, Meij P, Vervoort MB, et al. Direct immunosuppressive effects of EBV-encoded latent membrane protein 1. J Immunol 2000;165(2):663-670.

120. Nanbo A, Kawanishi E, Yoshida R, Yoshiyama H. Exosomes derived from Epstein-Barr virus-infected cells are internalized via caveola-dependent endocytosis and promote phenotypic modulation in target cells. J Virol 2013;87(18):10334-10347.

121. Klibi J, Niki T, Riedel A, et al. Blood diffusion and Th1suppressive effects of galectin-9-containing exosomes released by Epstein-Barr virus-infected nasopharyngeal carcinoma cells. Blood 2009;113(9):1957-1966.

122. Meckes DG, Jr., Shair KH, Marquitz AR, Kung CP, Edwards RH, Raab-Traub N. Human tumor virus utilizes exosomes for intercellular communication. Proc Natl Acad Sci U S A 2010;107(47): 20370-20375.

123. Ansari MA, Singh VV, Dutta S, et al. Constitutive interferoninducible protein 16-inflammasome activation during EpsteinBarr virus latency I, II, and III in B and epithelial cells. J Virol 2013;87(15):8606-8623.

124. Baglio SR, van Eijndhoven MA, Koppers-Lalic D, et al. Sensing of latent EBV infection through exosomal transfer of 5'pppRNA. Proc Natl Acad Sci U S A 2016;113(5): E587-E596.

125. Chugh PE, Sin SH, Ozgur S, et al. Systemically circulating viral and tumor-derived microRNAs in KSHV-associated malignancies. PLoS Pathog 2013;9(7):e1003484.

126. Naranatt PP, Krishnan HH, Svojanovsky SR, Bloomer C, Mathur $\mathrm{S}$, Chandran B. Host gene induction and transcriptional reprogramming in Kaposi's sarcoma-associated herpesvirus 
(KSHV/HHV-8)-infected endothelial, fibroblast, and B cells: insights into modulation events early during infection. Cancer Res 2004;64(1):72-84.

127. Meckes DG, Jr., Gunawardena HP, Dekroon RM, et al. Modulation of B-cell exosome proteins by gamma herpesvirus infection. Proc Natl Acad Sci U S A 2013;110(31): E2925-E2933.

128. Kuo HT, Ye X, Sampaio MS, Reddy P, Bunnapradist S. Cytomegalovirus serostatus pairing and deceased donor kidney transplant outcomes in adult recipients with antiviral prophylaxis. Transplantation 2010;90(10):1091-1098.

129. McDevitt LM. Etiology and impact of cytomegalovirus disease on solid organ transplant recipients. Am J Health Syst Pharm 2006;63(19 Suppl. 5):S3-S9.

130. Roux A, Mourin G, Fastenackels S, et al. CMV driven CD8(+) Tcell activation is associated with acute rejection in lung transplantation. Clin Immunol 2013;148(1):16-26.

131. Plazolles N, Humbert JM, Vachot L, Verrier B, Hocke C, Halary F. Pivotal advance: The promotion of soluble DC-SIGN release by inflammatory signals and its enhancement of cytomegalovirusmediated cis-infection of myeloid dendritic cells. J Leukoc Biol 2011;89(3):329-342.

132. Halary F, Amara A, Lortat-Jacob H, et al. Human cytomegalovirus binding to DC-SIGN is required for dendritic cell infection and target cell trans-infection. Immunity 2002;17(5):653-664.

133. Ota M, Serada S, Naka T, Mori Y. Expression of MHC class I molecule in HHV-6B-infected cells. Microbiol Immunol 2014;58(2):Februarycover.

134. Wang X, Wang HK, Li Y, et al. microRNAs are biomarkers of oncogenic human papillomavirus infections. Proc Natl Acad Sci U S A 2014;111(11):4262-4267.

135. Honegger A, Schilling D, Bastian S, et al. Dependence of intracellular and exosomal microRNAs on viral E6/E7 oncogene expression in HPV-positive tumor cells. PLoS Pathog 2015;11(3): e1004712.

136. Melo SA, Sugimoto H, O'Connell JT, et al. Cancer exosomes perform cell-independent microRNA biogenesis and promote tumorigenesis. Cancer Cell 2014;26(5):707-721.
137. Feng Z, Hensley L, McKnight KL, et al. A pathogenic picornavirus acquires an envelope by hijacking cellular membranes. Nature 2013;496(7445):367-371.

138. Feng Z, Li Y, McKnight KL, et al. Human pDCs preferentially sense enveloped hepatitis A virions. J Clin Invest 2015;125(1): 169-176.

139. Chai N, Chang HE, Nicolas E, Han Z, Jarnik M, Taylor J. Properties of subviral particles of hepatitis B virus. J Virol 2008;82(16):7812-7817.

140. Ahsan NA, Sampey GC, Lepene B, et al. Presence of viral RNA and proteins in exosomes from cellular clones resistant to Rift Valley fever virus infection. Front Microbiol 2016;7:139.

141. Silvas JA, Popov VL, Paulucci-Holthauzen A, Aguilar PV. Extracellular vesicles mediate receptor-independent transmission of novel tick-borne Bunyavirus. J Virol 2016;90(2):873-886.

142. Lozach PY, Kuhbacher A, Meier R, et al. DC-SIGN as a receptor for phleboviruses. Cell Host Microbe 2011;10(1):75-88.

143. Varkey JB, Shantha JG, Crozier I, et al. Persistence of Ebola virus in ocular fluid during convalescence. N Engl J Med 2015;372(25): 2423-2427.

144. Uyeki TM, Erickson BR, Brown S, et al. Ebola virus persistence in semen of male survivors. Clin Infect Dis 2016;62: 1552-1555.

145. Atkinson B, Hearn P, Afrough B, et al. Detection of Zika virus in semen. Emerg Infect Dis 2016;22(5):940.

146. Malkki H. CNS infections: Zika virus infection could trigger Guillain-Barre syndrome. Nat Rev Neurol 2016;12(4):187.

147. Garcez PP, Loiola EC, Madeiro da Costa R, et al. Zika virus impairs growth in human neurospheres and brain organoids. Science 2016;352:816-818.

148. Williamson PR, Nash TE, Williamson KC, Nath A. CNS infections in 2015: emerging catastrophic infections and new insights into neuroimmunological host damage. Lancet Neurol 2016;15(1):17-19.

149. Han Z, Madara JJ, Liu Y, et al. ALIX rescues budding of a double PTAP/PPEY L-domain deletion mutant of Ebola VP40: a role for ALIX in Ebola virus egress. J Infect Dis 2015;212(Suppl. 2): S138-S145. 